\title{
Effects of an Adaptogen-Based Supplement on Stress Parameters in Healthy Volunteers
}

\begin{abstract}
The effects of an adaptogen-based dietary supplement on stress indicators and stress perception were evaluated in health volunteers using a double-bind, placebo-controlled protocol. Seventy-seven healthy 24-60 year old male and female participants were enrolled, with 74 completing the study. Participants were given a 28-day supply of the supplement or the corresponding placebo and instructed to take $1 \mathrm{oz}$ each morning $30 \mathrm{~min}$. before breakfast. The supplement was a proprietary blend of 10 adaptogens including Eleurtherococcus, Schizandra and Rhodiola extracts, plus B vitamins. On the morning of enrollment, the participants completed a stress perception survey, had blood samples drawn for a highly sensitive C-reactive protein (HS-CRP), cortisol and homocysteine, and returned to the clinic after 28 days to repeat the stress perception survey and blood tests. A $40 \%$ reduction $(p<0.05)$ in the number of participants with abnormally elevated HS-CRP in the supplement group compared to the placebo group was observed. Furthermore, there was a 30\% reduction in levels of HSCRP in the supplemented group relative to the placebo group. Only one of the 5 subjects $(20 \%)$ with abnormal (elevated) serum homocysteine levels remained elevated after 4 weeks of supplementation. In contrast, 4 of the $6(66 \%)$ subjects in the control group remained elevated after 4 weeks. Five stress perception parameters were significantly reduced $(10-20 \%)$ in the supplemented group compared to two parameters in the placebo group. The results of this study indicate that the adaptogen/vitamin blend is effective at reducing subjective (perception survey) and objective (HS-CRP) stress measures compared to the placebo.
\end{abstract}

Keywords: Adaptogens; Stress; C-reactive protein; Homocysteine; B vitamins
Research Article

Volume 10 Issue 1 - 2017

Mohd Shara ${ }^{1 *}$, Eyad Kakish ${ }^{2}$ and Sidney J

Stohs ${ }^{2}$

${ }^{1}$ Faculty of Pharmacy, Jordan University of Science and

Technology, Jordan

${ }^{2}$ Creighton University Medical Center, USA

*Corresponding author: Mohd Shara, Department of Clinical Pharmacy, Chair, King Abdullah University Hospital and Clinics, Jordan University of Science and Technology, P.O. Box 3030, Iribd 22110, Tel: Cell: 962-79-753-7770;

Email: maalhatamleh@just.edu.jo

Received: November 20, 2017 | Published: December 12, 2017

\section{Introduction}

According to the American Institute of Stress, over $70 \%$ of people regularly experience physical and/or psychological symptoms caused by stress. The primary causes of stress in decreasing order of occurrence are: job pressure, money, health, relationships, poor nutrition, media overload, and sleep deprivation [1]. Persistent stress leads to fatigue, irritability, insomnia, weakened immune system, and increased susceptibility various diseases including cardiovascular diseases. Annual cost to employers associated with missed work and stress-related health care in the USA is estimated to be over three billion dollars [1].

The role of oxidative stress and vascular inflammation in cardiovascular disease is well known [2]. Biomarkers of oxidative stress and inflammation help identify patients at risk for cardiovascular disease [3], although there is not a single biomarker that can estimate absolute risk of future events. Biomarkers exist for assessing oxidative stress, antioxidant levels, pro-inflammatory cytokines, anti-inflammatory cytokines, and chemokines [3].

Oral nutrition in the form of dietary supplements is consumed by70 \% of the general population on a daily basis, including the use of supplements for the management of stress. Studies indicate that approximately $70-90 \%$ of health care professionals including physicians and nurses recommend dietary supplements [4,5], while $64-69 \%$ of the general population use dietary supplements [6].

Adaptogens are defined as herbal extracts or preparations that increase tolerance to stressors, enhance attention and mental endurance, increase tolerance to mental fatigue and physical exhaustion, and reduce stress-induced disorders related to the immune and neuro-endocrine systems [7-9]. Examples of plants that have been studied for their anti-stress activities include Rhodiola, Schizandra, Eleutherococcus, Withania, Emblica and Glycyrrhiza [7,9-13].

Historically, the term "adaptogen" was introduced into the scientific literature by N. Lazarev in 1957 who referred to plantderived substances that increased "the state of non-specific resistance". For detailed historical reviews see Panossian and Wagner $[8,9]$. Because the pharmacological profiles of plants used for their adaptogenic properties vary widely, combinations of plant extracts are commonly used in the preparation of adaptogenic products $[9,14,15]$. The purpose of this study was to evaluate the effects of an adaptogen/vitamin-based supplement on stress perception and stress indicators in healthy volunteers after 28 days of supplementation vs. placebo. 


\section{Materials and Methods}

The study was double-blinded and placebo-controlled. A total of 77 healthy 24-60 year old male and female participants were enrolled of which 74 completed the study. The protocol was approved by the Creighton University Institutional Review Board, Omaha, NE USA. Prospective subjects were recruited through advertisements in the local paper and on the Creighton University campus.

The supplement consisted of a liquid formula composed of B vitamins, betaine, and a proprietary water/ethanol extract blend derived from 10 adaptogenic plants including Eleutherococcus sensicosus, Schizandra chinensis, Rhodiola rosea, Glycyrrhiza uralensis, Crataegus oxyacantha and Aralia manchurica (AdvoCare International, Plano, TX). A one ounce serving of the extract contained 2 grams of the extract standardized for its total polyphenolic content. The composition of the formulation is presented in Table 1. The placebo contained the same appearance, flavor and all ingredients except the vitamins, betaine and adaptogen blend.

Table 1: Liquid adaptogen-vitamin formula supplements facts.

\begin{tabular}{|c|c|c|}
\hline & $\begin{array}{l}\text { Amount } \\
\text { Per Serving }\end{array}$ & $\%$ DV \\
\hline Calories & 15 & \\
\hline Total Carbohydrates & $3 g$ & $1 \%$ \\
\hline Sugars & $3 g$ & \\
\hline Riboflavin & $3.4 \mathrm{mg}$ & $200 \%$ \\
\hline Niacin (as niacinamide) & $40 \mathrm{mg}$ & $200 \%$ \\
\hline Vitamin $\mathrm{B} 6$ (as pyridoxine $\mathrm{HCl}$ ) & $4 \mathrm{mg}$ & $200 \%$ \\
\hline Folic acid & $800 \mathrm{mcg}$ & $200 \%$ \\
\hline Vitamin B12 (as cyanocobalamin) & $500 \mathrm{mcg}$ & $8333 \%$ \\
\hline Pantothenic acid (as calcium pantothenate) & $20 \mathrm{mg}$ & $200 \%$ \\
\hline Betaine & $100 \mathrm{mg}$ & \\
\hline $\begin{array}{c}\text { Proprietary blend of Eleutherococcus } \\
\text { senticosus } \\
\text { (leaf, stem, root), Schisandra chinensis } \\
\text { (seed), } \\
\text { Aralia manchurica (flower), Crataegus } \\
\text { oxyacantha } \\
\text { (leaf), Viburnum sargenti (leaf, berry), } \\
\text { Glycyrrhiza } \\
\text { uralensis (root), Rhaponticum } \\
\text { carthamoides (root), } \\
\text { Rhodiola rosea (flower), Sorbus aucuparia } \\
\text { (whole } \\
\text { plant), Iconotus obliquus (root) } \\
\text { (ther } \\
\text { natural flavors. Beet root extract, citric } \\
\text { acid, glycerin, potassium sorbate (as } \\
\text { preservative), sodium benzoate (as } \\
\text { preservative), xanthan gum, and sucralose. }\end{array}$ & $2 g$ & \\
\hline
\end{tabular}

All participants completed a stress perception survey (Table 2) on the morning of enrollment and blood samples were drawn for highly sensitive C-reactive protein (HS-CRP), cortisol and homocysteine. The analyses of these biomarkers were conducted by Physicians Laboratory, a commercial laboratory (Omaha, Nebraska USA). The participants were randomized into two groups, and were given a 28-day supply of the supplement labeled either A or B, and instructed to take one ounce of the product each morning 30 min before breakfast. After 28 days, the participants completed the same survey and provided blood samples for the same three analytical tests.

Table 2: Stress level survey.

\begin{tabular}{|c|c|c|}
\hline 1 & Things must be perfect & 12345 \\
\hline 2 & I must do it myself & 12345 \\
\hline 3 & I feel more isolated from my family or close friends & 12345 \\
\hline 4 & I feel that people should listen better & 12345 \\
\hline 5 & I am not where I want to be in life & 12345 \\
\hline 6 & I must not fail & 12345 \\
\hline 7 & $\begin{array}{l}\text { When overworked, I cannot say "no" to new } \\
\text { demands without guilt }\end{array}$ & 12345 \\
\hline 8 & I avoid being alone & 12345 \\
\hline 9 & I feel increasingly cynical and disenchanted & 12345 \\
\hline 10 & I am unable to laugh at a joke about myself & 12345 \\
\hline 11 & I avoid speaking my mind & 12345 \\
\hline 12 & I have trouble getting to sleep & 12345 \\
\hline 13 & I automatically express negative attitudes & 12345 \\
\hline 14 & $\begin{array}{l}\text { I seem further behind at the end of the day than } \\
\text { when I started }\end{array}$ & 12345 \\
\hline 15 & $\begin{array}{l}\text { I forget deadlines, appointments and personal } \\
\text { possessions }\end{array}$ & 12345 \\
\hline 16 & $\begin{array}{l}\text { I am irritable, short-tempered, disappointed in the } \\
\text { people around me }\end{array}$ & 12345 \\
\hline 17 & Sex seems like more trouble than it's worth & 12345 \\
\hline 18 & I consider myself exploited & 12345 \\
\hline 19 & I feel dissatisfied with my personal life & 12345 \\
\hline 20 & I feel unrested. & 12345 \\
\hline
\end{tabular}

All data were analyzed for statistical significance between the groups receiving the placebo versus the adaptogen/vitamin supplement for 28 days using a paired 2-tailed t-test. Data are expressed as the mean with the standard deviation. Differences with a $\mathrm{p}<0.05$ are considered significant.

\section{Results}

The effects of 28 day supplementation with the adaptogen/ vitamin formulation on blood levels of HS-CRP, homocysteine and cortisol are presented in Table 3. Daily supplementation for 
28 days significantly reduced the HS-CRP by approximately 30 $\%$ as compared to the placebo control group. Daily use of the adaptogen/vitamin formulation resulted in a non-significant 8 $\%$ decrease in blood homocysteine levels and no effect on blood cortisol.

Table 3: Effect of 28 day adaptogen/vitamin supplement vs placebo on blood HS-CRP, homocysteine and cortisol levels.

\begin{tabular}{|c|c|c|c|c|}
\hline \multicolumn{2}{|c|}{ Group } & HS-CRP & Homocysteine & Cortisol \\
\hline \multirow{2}{*}{ Treated } & Pre- & $4.81 \pm 7.56$ & $8.87+2.40$ & $11.71 \pm 5.13$ \\
\cline { 2 - 5 } & Post- & $3.40 \pm 5.42$ & $8.16 \pm 1.97$ & $14.64 \pm 4.91$ \\
\hline \multirow{2}{*}{$\%$ Change } & Post- & $30 \% \downarrow$ & $8 \% \downarrow$ & $25 \% \downarrow$ \\
\hline \multirow{2}{*}{ Placebo } & Placebo & $4.81 \pm 7.56$ & $8.87+2.40$ & $11.71 \pm 5.13$ \\
\cline { 2 - 5 } & Post- & $5.70 \pm 8.21$ & $9.15 \pm 3.18$ & $13.48 \pm 4.42$ \\
\hline \multirow{2}{*}{$\%$ Change } & Post- & $18 \% \downarrow$ & $3.2 \% \downarrow$ & $15 \% \downarrow$ \\
\hline
\end{tabular}

The data are also expressed based on the change in the number of participants with abnormal laboratory values before and after 28 day treatment with the supplement or placebo control. As can be seen in Table 4 , a $40 \%$ decrease occurred in the number of participants with elevated HS-CRP after treatment as compared to pre-treatment with the adaptogen/vitamin formulation. An $80 \%$ decrease occurred in the number of treated subjects with elevated homocysteine after 28 days of treatment while a $33 \%$ decrease occurred in the placebo group. No changes occurred in the number of participants with elevated blood cortisol after treatment with the adaptogen/vitamin supplement or in the placebo group.

Table 4: Effect of 28 day adaptogen/vitamin supplementation on number of participants with elevated lab values.

\begin{tabular}{|c|c|c|c|c|}
\hline \multicolumn{5}{|c|}{ Number of Elevated/Abnormal Laboratory Values } \\
\hline \multirow{2}{*}{ Group } & HS-CRP & Homocysteine & Cortisol \\
\hline \multirow{2}{*}{ Treated } & Pre- & 10 & 5 & 2 \\
\cline { 2 - 5 } & Post- & 6 & 1 & 2 \\
\hline \multirow{2}{*}{$\%$ Decrease } & Post- & $40 \%$ & $80 \%$ & $0 \%$ \\
\hline \multirow{2}{*}{ Placebo } & Pre- & 17 & 6 & 1 \\
\cline { 2 - 5 } & Post- & 16 & 4 & 1 \\
\hline \% Decrease & Post- & $5.90 \%$ & $33 \%$ & $0 \%$ \\
\hline
\end{tabular}

A stress perception questionnaire was administered on day 0 and day 28 of the study (Table 5). Five stress perception scores (questions 3, 6, 7, 9, and 10) were significantly decreased (10-20 $\%$ ) in the participants supplemented with the adaptogen/vitamin product while two parameters were significantly reduced in the placebo group (questions 6 and 10).
Table 5: Effects of 28 day adaptogen/vitamin supplement vs. placebo on stress survey.

\begin{tabular}{|c|c|c|c|c|c|c|}
\hline \multicolumn{7}{|c|}{ Survey Parameter Mean Score } \\
\hline \multirow{2}{*}{ Group } & & Q3 & Q6 & Q7 & Q9 & Q10 \\
\hline \multirow{2}{*}{ Treated } & Pre- & 2.6 & 3.7 & 3.4 & 2.9 & 2.5 \\
\cline { 2 - 7 } & Post- & $2.2^{*}$ & $3.3^{*}$ & $2.9 *$ & $2.3^{*}$ & $2.0^{*}$ \\
\hline \multirow{2}{*}{ \% Decrease } & Post- & $15.4 \%$ & $10.8 \%$ & $14.7 \%$ & $20.7 \%$ & $20.0 \%$ \\
\hline \multirow{2}{*}{ Placebo } & Pre- & 2.3 & 3.7 & 3.7 & 2.9 & 2.5 \\
\cline { 2 - 7 } & Post- & 2.2 & $3.3 *$ & 3.2 & 2.5 & $2.0 *$ \\
\hline \% Decrease & Post- & $4.30 \%$ & $10.8 \%$ & $13.5 \%$ & $13.8 \%$ & $20.0 \%$ \\
\hline
\end{tabular}

\section{Discussion}

The most remarkable finding of this study was the $30 \%$ reduction in HS-CRP blood levels in response to adaptogen/ vitamin ingestion for 28 days relative to the placebo control (Tables 3), while a $40 \%$ decrease occurred in the number of treated subjects with elevated HS-CRP (Table 4). Furthermore, the survey findings indicated that the adaptogen /vitamin preparation reduced stress perceptions to a greater extent than the corresponding placebo (Table 5). Elevated HS-CRP is strongly associated with an increased incidence of heart attack, stroke, type 2 diabetes and metabolic syndrome [2,12,16-19], and the combination product used in this study significantly reduced these levels.

Hyperhomocysteinemia is associated with an increased risk of cardiovascular disease, diabetes, osteoporosis, Alzheimer's disease, other dementias, and is also believed to be a marker of pathological oxidant stress [20]. The administration of the adaptogen/vitamin blend resulted in a non-significant decrease in blood homocysteine levels (Table 3), although the number of subjects with elevated homocysteine levels at the start of treatment decreased by $80 \%$ after 28 days of product ingestion (Table 4). Ingestion of B vitamins as vitamin B12, folate and vitamin B6 (pyridoxine) as present in the product used in this study have been shown to result in decreased blood homocysteine levels [21]. As a consequence, it is not clear whether the decrease in homocysteine in the current study was due to the B vitamins or the adaptogen blend.

Cortisol levels are another indicator of stress [22], and decreases in serum cortisol are believed to be produced by at least some adaptogens [23]. For example, the adaptogen ashwagandha (Withania) significantly reduced stress in adults and significantly decreased serum cortisol levels after 60 days of treatment [24]. The reason for the lack of an effect of the adaptogen/vitamin product on cortisol levels after 28 days of treatment is not known. The product did not contain ashwagandha.

A rapidly growing body of research literature supports the concept that inflammation plays a pivotal role in cardiovascular 
and metabolic diseases $[2,12,16-18,25]$. The anti-inflammatory and stress protective effects of adaptogens are believed to be associated with the homeostatic regulation of several different pathways and mechanisms including the hypothalamic-pituitaryadrenal axis, the neuro-endocrine-immune complex, and key stress response mediators [11,23,26]. The pharmacological profile of adaptogens has been summarized by Panossian \& Wagner [9].

Up-regulation of the stress sensor heat shock protein (Hsp) 70 by adaptogenic substances results in the inhibition of the expression of nitric oxide (NO) synthase while concurrently enhancing mitochondrial antioxidant activity, glutathione synthesis, and ATP generation [23,26]. Hsp 70 also interacts directly with glucocorticoid receptors and controls key mediators of stress as Jun N-terminal protein kinase (JBK1), and the transcription factor forkhead box 0 (FOXO). A more recent study by Panossian et al. [15] has shown that an adaptogenic blend of extracts from Eleutherococcus, Shizandra and Rhodiola, constituents present in the adaptogenic blend used in this study, stimulated the expression of the stress hormone neuroprotein Y (NPY) and heat shock protein (Hsp) 72. The net result of these mechanistic effects is an increase in mental and physical performance and possibly longevity $[11,23,26]$. The chemical substances believed to be responsible for adaptogenic properties include phenylpropanoids, phenethylamines and tetracyclic triterpenoids $[9,11]$. Because of the widely diverse chemical nature of adaptogens and their equally wide distribution throughout the plant kingdom, combinations of adaptogenic plant extracts (Table 1) are commonly used in order to ensure a broad incorporation of active constituents $[9,11,14,15]$.

\section{Conclusion}

The use of the adaptogen/vitamin product for 28 days resulted in a significant objective decrease of the stress biomarker HS-CRP, a non-significant decrease in homocysteine, and decreases in the numbers of subjects with elevated HS-CRP and homocysteine. Furthermore, significant subjective decreases in stress were observed in response to the adaptogen/vitamin blend based on a perception survey.

\section{Acknowledgment}

None.

\section{Conflict of Interest}

This study was supported by a grant from AdvoCare International, Plano, TX. SJS has served as a consultant for AdvoCare. MS and EK have no perceived conflicts of interest.

\section{References}

1. Anon (2017) Stress statistics.

2. Siti HN, Kamisah Y, Hamisiah J (2015) The role of oxidative stress, antioxidants and vascular inflammation in cardiovascular disease (a review). Vascul Pharmacol 71: 40-56.

3. Stoner L, Lucero AA, Palmer BR, Jones LM, Young JM, et al. (2013) Inflammatory biomarkers for predicting cardiovascular disease. Clin Biochem 46(15): 1353-1371.
4. Dickinson A, Boyon N, Shao A (2009) Physicians and nurses use and recommend dietary supplements: report of a survey. Nutr J 8: 29.

5. Dickinson A, Shao, A, Boyon N, Franco JC (2011) Use of dietary supplements by cardiologists, dermatologists and orthopedists: report of a survey. Nutr J 10: 20.

6. Dickinson A, Blatman J, El-Dash N, Franco JC (2014) Consumer usage and reasons for using dietary supplements: report of a series of surveys. J Amer Coll Nutr 33(2): 176-182.

7. Wagner HKM (1995) Immunostimulants and Adaptogens from Plants. Phytochemistry of Medicinal Plants, JT Arnason (Ed.), Plenum Press, New York, USA, p. 1-18.

8. Panossian A, Wagner H (2005) Stimulating effect of adaptogens: An overview with particular reference to their efficacy following single dose administration. Phytother Res 19(10): 819-839.

9. Panossian A, Wagner H (2011) Adaptogens: A review of their history, biological activity, and clinical benefits. HerbalGram 90: 52-63.

10. Chen TS, Liou SY, Chang YL (2008) Antioxidant evaluation of three adaptogen extracts. Amer J Chin Med 35(6): 1209-1217.

11. Panossian A (2013) Adaptogens in mental and behavioral disorders. Psychiat Clin North Amer 36(1): 49-64.

12. Recio MC, Giner RM, Manez S (2016) Immunomodulatory and antiproliferative properties of Rhodiola species. Planta Med 82(1112): 952-960.

13. Anshakova VV, Stepanova AV, Uvarov DM, Naumova KN, Vasiliev PP (2016) Adaptogenic activity of a complex biomedication based on a northern renewable raw material. Wiad Lek 69(1 Pt 2): 55-60.

14. Seely D, Singh R (2007) Adaptogenic potential of a polyherbal natural health product: report on a longitudinal clinical trial. Evid Based Complement. Alternat Med 4(3): 375-380.

15. Panossian A, Wikman G, Kaur P, Asea A (2012) Adaptogens stimulate neuropeptide $Y$ and $H s p 72$ expression and release in neuroglia cells. Front Neurosci 6: 6 .

16. Sutherland JP, McKinley, Eckel RH (2004) The metabolic syndrome and inflammation. Metab Syndr Disord 2(2): 82-104.

17. Ndumele CE, Pradhan AD, Ridker PM (2006) Interrelationships between inflammation, C-reactive protein, and insulin resistance. J Cardiometab Syndr 1(3): 190-196.

18. Pfutzner A, Schondorf T, Hanefeld M, Frost T (2010) High-sensitivity C-reactive protein predicts cardiovascular risk in diabetic and nondiabetic patients: effects of insulin-sensitizing treatment with proglitazone. J Diabetes Sci Technol 4(3): 706-716.

19. Windgasser EB, Funtowicz L, Lundsford TN, Harris LA, Mulvagh SL (2011) C-Reactive protein and high-sensitivity C-reactive protein: an update for clinicians. Postgrad Med 123(1): 114-119.

20. Hoffman M (2011) Hypothesis: hyperhomocysteinemia is an indicator of oxidant stress. Med Hypothesis 77(6): 1088-1093.

21. Huang T, Chen Y, Yang B, Yang J, Wahlqvist ML, et al. (2012) Metaanalysis of B vitamin supplementation on plasma homocysteine cardiovascular and all-cause mortality. Clin Nutr 31(4): 448-454.

22. Lucassen EA, Cizza G (2012) The hypothalamic-pituitary-adrenal axis, obesity, and chronic stress exposure: sleep and HPA axis in obesity. Curr Obes Res 1(4): 208-215.

23. Panossian A, Wikman G (2009) Evidence-based efficacy of adaptogens in fatigue, and molecular mechanisms related to their stress-protective activity. Curr Clin Pharmacol 4(3): 198-219. 
24. Chandrasekhar K, Kapoor J, Anishetty S (2012) A prospective randomized double-blind, placebo-controlled study of safety and efficacy of a high-concentration full-spectrum extract of ashwagandha root in reducing stress and anxiety in adults. Indian Psychol Med 34(3): 255-262.
25. Calabro P, Golia E, Yeh EY (2009) CRP and the risk of atherosclerotic events. Semin Immunopathol 31(1): 79-94.

26. Panossian A, Wikman G (2006) Effects of adaptogens on the central nervous system and the molecular mechanisms associated with their stress-protective activity. Pharmaceuticals (Basel) 3(1): 188-224. 